\title{
53 Years Old is A Reasonable Cut-Off Value To Define Young And Old Patients In Clear Cell Renal Cell Carcinoma:A Study Based On TCGA And SEER Database
}

\section{Fucai Tang}

Guangdong Provincial Work Injury Rehabilitation Center

\section{Zechao Lu}

First Clinical College of Guangzhou Medical University

\section{Chengwu He}

Department of Urology, The Eighth Affiliated Hospital, Sun Yat-sen University

\section{Hanbin Zhang}

The Second Clinical College of Guangzhou Medical University

\section{Weijia Wu}

Department of Urology, The Eighth Affiliated Hospital, Sun Yat-sen University

Zhaohui He ( $\nabla$ gzgyhzh@163.com )

The Eighth Affiliated Hospital, Sun Yat-sen University https://orcid.org/0000-0003-2289-9603

\section{Research article}

Keywords: Age; age-related genes; Clear cell renal cell carcinoma; The Cancer Genome Atlas; Surveillance Epidemiology and End Results

Posted Date: August 14th, 2019

DOI: https://doi.org/10.21203/rs.2.12816/v1

License: (c) (i) This work is licensed under a Creative Commons Attribution 4.0 International License. Read Full License

Version of Record: A version of this preprint was published at BMC Cancer on May 29th, 2021. See the published version at https://doi.org/10.1186/s12885-021-08376-5. 


\section{Abstract}

Background The objectives of this study were to screen out cut-off age value and age-related differentially expressed genes (DEGs) in clear cell renal cell carcinoma (CCRCC) from Surveillance Epidemiology and End Results (SEER) database and The Cancer Genome Atlas (TCGA) database. Methods We selected 45,974 CCRCC patients from SEER and 530 RNA-seq data from TCGA database. The age cut-off value was defined using the X-tile program. Propensity score matching (PSM) was used to balance the differences between young and old groups. Hazard ratio (HR) was applied to evaluate prognostic risk of age in different subgroups. Age-related DEGs were identified via RNA-seq data. Survival analysis was used to assess the relationship between DEGs and prognosis. Results In this study, we divided the patients into young $(n=14276)$ and old $(n=31698)$ subgroups according to cut-off value (age=53). Age $>53$ years was indicated as independent risk factor for overall survival (OS) and cancer specific survival (CSS) of CCRCC before and after PSM. The prognosis of old group was worse than that in young group. 176 age-related DEGs were obtained by 530 CCRCC RNA-seq analysis result. The expression levels of KCNE5, CLDN6 and CACNG6 were related to prognosis of the elderly. Conclusion 53 years old was cut-off value in CCRCC. The prognosis of the elderly was worse than young people. KCNE5, CLDN6 and CACNG6 were age-related differential genes which might play an important role in the poor prognosis of elderly CCRCC patients.

\section{Background}

Over the past two decades, the incidence of renal cell carcinoma (RCC) at every stages was increased and this situation resulted in a steady increase in mortality per unit of population [1]. It is estimated that 65340 Americans will be diagnosed with RCC, and 14970 Americans will die of this cancer in 2018. RCC comprises about $3.8 \%$ of all new cancer. And the median age of RCC patient is 64 ages old. Clear cell renal cell carcinoma (CCRCC) is the most common subtype of RCC, it accounts for about $80 \%$ of RCC [2]. Age has prognostic significance in many solid cancers, and one of renal cancer known risk factors is age $[3,4]$. RCC shows a more favorable prognosis in young patiens, which may be due to the lower state of diagnosis [5]. In addition, age can influence the structural and molecular properties of the tumor vasculature in CCRCC by comparing the vascular properties of patients who over the age of 65 and under 65 years old [6]. Furthermore, expression levels of Piwil 1 mRNA in patients who under 64 years old are higher than that in older people ( $>64$ years old). But there still is no optimal age cut off value to define elderly and young people in CCRCC. Therefore, we determined the optimal cut-off value for age analyzing the clinical data SEER database, and explored differentially expressed genes (DEGs) between older and younger people of CCRCC by analyzing RNA-seq data from TCGA in present study.

\section{Methods}

\section{Study population from SEER}


SEER Stat software(version 8.3.5) was used to download CCRCC clinical data from the National Cancer Institute's Surveillance, Epidemiology, and End Results (SEER) database. The downloaded data included: patient ID, the year and age at the time of diagnosis, sex, race, histological type, survival time, tumor size, marital status, Grade, SEER historic stage A, and cuase of death.

CCRCC patients were selected according to the following criteria: (1) site record International Classification of Diseases for Oncology, Third Revision (ICD-0-3) was C649; (2) histological type was 8310/3; (3) the year at time of diagnosis was 1988-2014. (4) CCRCC was primary tumor. The exclusion criteria were listed as following: (1) patients without race and gender information; (2) patients whose tumor size, survival time and other clinical information we need in this study were unknown.

\section{Variable declaration}

Race was defined as white, black and other. Marital was divided into Single/Other, and married. Tumor size was divided into less than $4 \mathrm{~cm}, 4 \mathrm{~cm}$ to $7 \mathrm{~cm}$, and greater than $7 \mathrm{~cm}$. Grade was grouped as I, II, III, IV. Laterality was divided into left and right. The SEER historic stage options included localized, regional and distant. And the chemotherapy, radiotherapy were divided into yes or no.

\section{Cut off age in CCRCC}

The "x tile plot" can provide a single, global assessment of every possible way by dividing a population into low- and high-level marker expression [7]. We used X-tile plots to assessed all possible age cutoff value, and the survival at every age cutoff value was computed by the log rank test. Then the most appropriate cut-off value was selected which had the highest X2 value.

\section{RNA-seq Analysis of CCRCC from TCGA}

The RNA sequencing and clinical information of CCRCC were download from TCGA database. We used these RNA-seq data for DEGs screening between younger and older group by edgeR package (adjusted $p$ value $<0.05$ and $\mid \log 2$ fold change (FC) $\mid \geq 1$ ). Then, we extracted clinical data from older adults $(>53$ years), including survival time and survival status. We selected the DEGs from the small to large false discovery rate (FDR). And the top 10 genes was for survival analysis. The differentially expression levels of these top 10 genes in these old patients were obtained. The median of gene expression was used to classify low and high group. Log rank test was used to compare statistically significant differences between high and low expression groups.

\section{Statistical Analysis}

We divided the patients into young and old groups according to the X-tile's best cut-off value. Chi-square test was used to compare the differences in the distribution of variables between younger and older group. We calculated the overall survival (OS) and cancer specific survival (CSS). In the CSS calculation, the cause of death for other reasons was defined as censorship. Propensity score matching (PSM) used logistic regression included relevant variables of Sex, race, marital status, size, grade, SEER historic stage 
A, radiation and chemotherapy to balance the baseline differences between the younger and older groups. The OS and CSS Survival curves were generated using the Kaplane-Meier method. And univariate and multivariate analysis Cox regression models were applied to adjust prognostic variables. The cases were stratified according to the relevant variables. Hazard Ratio (HR) of the CSS was calculated according to the age. When the 2-sided $P$ value was $<0.05$, the differences were considered statistically significant. The SPSS 24.0 and R 3.4.3 were used to conduct statistical analysis and DEGs screening.

\section{Results}

\section{3 was the age cut-off value and Baseline Characteristics}

We obtained 45,974 CCRCC patients in totally. The median age of these patients was 60 years old (interquartile range: 51-69). At the same time, $X$ tile result showed that 53 years old was defined as the best cut-off value for age (Figure 1). Then we divided the cohort into two groups: younger group (53 years or younger), older group (older than 53 years) according to the cut-off value. The detailed features of the patients between the two groups were presented in Table 1 (Table 1).

\section{Survival Analysis}

In the young group, 5- and 10 -year OS rates were $86.4 \%$ and $78.2 \%$ respectively. In the old group, 5 - and 10-year OS rates were $72.8 \%$ and 54.5 respectively $(P<0.001$; Figure $2 \mathrm{~A})$. Univariate analysis results indicated that age, sex, race, marital status, size, grade, laterality, SEER historic stage $A$, radiation and chemotherapy,could predict patient suvival outcomes. Meanwhile, multivariate analysis showed that the age, sex, race, marital status, size, grade, laterality, SEER historic stage A, radiation and chemotherapy were independent prognostic factor for CCRCC OS(Table 2).

In the young group, 5- and 10-year CSS rates were $89.4 \%$ and $81.7 \%$ respectively. In the old group, 5 - and 10-year CSS rates were $84.3 \%$ and $72.8 \%$ respectively $(P<0.001$; Figure $2 \mathrm{~B})$. The results of univariate analysis showed that age, sex, marital status, size, grade, laterality, SEER historic stage A, radiation and chemotherapy were associated with patient's prognosis. Multivariate analysis showed that age, marital status, size, grade, SEER historic stage A, radiation and chemotherapy were independent prognostic factors for CCRCC CSS(Table 3).

\section{Survival Analysis after PSM}

The clinical characteristics of the patients between the younger and older groups had obvious differences. So PSM method was applied to balance the differences between the variables, and generated a new queue (All covariates were well balanced, $P$ values $>0.05 ;$ Table 1 ). Univariate analysis results showed that HR for OS of the older patient were 2.056 (95\% Cl:1.948-2.170; $P<0.001)$, HR for CSS were 1.496(95\% Cl, 1.399-1.600; $P<0.001)$ when compared with the younger group. In the PSM queue, the younger people also had a higher survival rate than older people (Figure $2 \mathrm{C}, \mathrm{D}$ ). Multivariate analysis results showed that compared with the younger group , HR for OS of the older patient were 2.128 
$\varangle 95 \% \mathrm{Cl}: 2.015-2.247 ; P<0.001 \rrbracket, \mathrm{HR}$ for CSS were 1.573(95\%Cl:1.470-1.682; $P<0.001)$. Other variable results were showed in Table 2, 3 .

\section{Subgroup analysis}

We performed a subgroup analysis based on sex, race, marital status, size, grade, laterality, SEER historic stage A, radiation, and chemotherapy. In most subgroups, the older group had a worse prognosis than the younger group. However radiation, and chemotherapy and prognostic differences between young and old groups were not statistically significant $(P>0.05)$ (Figure 3 ).

\section{Three gene associated with poor prognosis in the elderly}

We finally got 176 DEGs included 114 up-regulated genes and 62 down-regulated genes (Table S1). By ranking the FDR, we selected the top 10 gene(Table 4) for survival analysis, and investigated the correlation between the expression of these genes and prognosis of the elderly. The results showed that

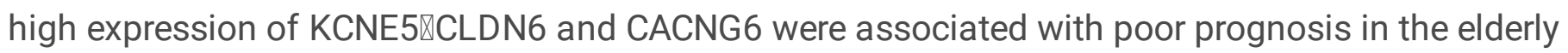
(Figure 4).

\section{Discussion}

A total of 45,974 CCRCC patients were included in the SEER database, of which the 53-year-old cut-off value was used to divide the younger and older groups. Survival analysis results showed that younger age (under age 53) was an independent predictor of CCRCC. And we obtained some genes related to old patients with CCRCC by analyzing the RNA-seq data downloaded from TCGA.

Some studies reported that the 40 years old was suitable to act as the dividing line between young and old CCRCC patients. Xavier Taccoen et al. found that young (under 40 years of age) age was an independent prognostic factor for CCRCC, with a better prognosis [8]. Atiqullah Aziz et al found that young patients with RCC (age 40 or under) have a significantly lower all cause and disease specific killed [9]. Ho Won Kang et al. also found that young age was associated with favorable pathological features, although it is not survival independent prognostic factors in surgically treated RCC patients. But the result of the Kaplan-Meier analysis showed that the CCS rate was significantly better in the young age group than the other groups (middle age: $\geq 4$ and $<60$ years; old age: $\geq 60$ years) [10]. Analysis results between younger and older RCC patients (20-39 and 40-79) of Jeong Ho Kim et al found that younger RCC patients would have more favorable histological subtypes. And the 5-year CSS rates for young and older patients were $95.5 \%$ and $90.5 \%$ respectively. However, after PSM, the five-year CSS rate was $95.5 \%$ for the younger group and $94.7 \%$ for the older group, and the prognosis was not significantly different ( $\log$ rank $p=0.184$ ) [11]. In addition to the 40 years old, there were many age cut-off values, such as 45 and 55 years old. Yoshinobu Komai et al used the 45-year-old as a cut-off value for younger and older group. Compared with the older patients, the young patients with RCC had similar recurrence-free survival rates but better CSS rates [12]. Eun-Jung Jung et al believed that younger age was an independent predictor of prognosis through multivariate analysis. Whereas in their study, younger than 55 years of age 
was considered as young in CCRCC [13]. In this study, we used X-tile plots to assessed all possible age cuttoff value, and finally selected the age 53 as the cut off value for dividing younger and older group. And younger groups had better OS and CSS compared to older groups. What's more, in the subgroups anaysis, The prognosis of old group was worse than that of young group in all subgroups of this study, especially in the Dmax $<=4 \mathrm{~cm}$ subgroups (HR = 3.710(3.006-4.579), $P<0.001)$.

In recent years, with the development of cancer gene sequencing and targeted therapies, the research on gene expression of CCRCC had made some progress. In the CCRCC age-related studies, Xp11 translocation renal cell carcinoma was kind of RCC subtype, Malouf GG et al used the targeted therapy to treat patients, the objective responses was achieved and the patients got the better progression-free survival [14]. Mitchell TJ et al. analyzed the entire genome of CCRCC and found that $36 \%$ of patients experience $3 p$ loss and $5 q$ gain, which usually occured during childhood or adolescence. Meanwhile hotspots of point mutations in the 5' UTR of TERT, targeting a MYC-MAX-MAD1 repressor associated with telomere lengthening [15]. Malouf GG reported that ASPSCR1-TFE3 might be the most aggressive among the transcription factor E3 fusion genes in RCC patients [16].

In this study, we obtained some DEGs by comparing RNA-seq data from younger and older CCRCC patients. Then, the top 10 genes were used for survival analysis. As showed in the result (Figure 4), the expression of KCNE5(Potassium Voltage-Gated Channel Subfamily E Regulatory Subunit 5), CLDN6(Claudin 6) and CACNG6 (Calcium Voltage-Gated Channel Auxiliary Subunit Gamma 6) were related to the survival of the elderly.

KCNE5 is belongs to the human KCNE gene family. This KCNE gene mutations are related to various pathophysiological states, and most obvious KCNE gene mutation occurs in the arrhythmia [17]. However, its current research on cancer had not been reported. CLDN6 is a family member of CLDNs, which encodes a component of tight junction strands[18]. Some study had showed that CLDN6 expression was low in breast cancer cells and closely related to the proliferation and invasion of the cells[19,20]. According to Gao F et al [21] there was a correlation between CLDN6 and gastric cancer. CLDN6 low expression has a certain negative impact on the prognosis of patients. Therefore, CLDN6 could be used as an important indicator for the prognosis of gastric cancer patients. But there was no research report on the role of CLDN6 in CCRCC. CACNG6 is a complete membrane protein. It stabilizes calcium channels during an inactive state [22]. CACNG6 polymorphism was associated with aspirin intolerance among asthma patients in the Korean population [23]. At the same time, our research also showed that KCNE5区 CLDN6 and CACNG6 were not only age-related DEGs for the elderly, but their expression levels were related to the survival of the elderly, so we hypothesized that these genes might have clinical monitoring value in CCRCC which deserved for further research.

Our study had several potential limitations. The leading known risk factors for renal cancer were smoking, obesity and hypertension [24-29]. However, due to the lack of corresponding data in the SEER database, we were unable to study these factors. At the same time, retrospective analyses always carried the risk of 
various biases. We used the subgroup, PSM analysis and incorporate large amounts of patients in this study to minimize potential biases.

\section{Conclusions}

In conclusion, we proposed that 53-year-old was a reasonable cut-off value among CCRCC patients, and the elderly group had a worse prognosis than the younger group. At the same time, we screened three age-related DEGs (GRIK1, CILP2 and CACNG6), which might be associated with poor prognosis in the elderly, but the specific mechanism remained to be further studied.

\section{Abbreviations}

CCRCC: clear cell renal cell carcinoma;

DEGs: Differentially expressed genes;

SEER: Surveillance Epidemiology and End Results;

TCGA: The Cancer Genome Atlas;

OS\overall survival;

CSS: cancer specific survival;

PSM: Propensity score matching;

HR: Hazard Ratio;

FC: fold change;

FDR $₫$ false discovery rate;

CPM『counts per million.

\section{Declarations}

\section{Funding}

The present study was supported by research start-up fee for the eighth affiliated hospital, Sun Yat-sen University (grant no.zdbykyqdf005); 2018 Provincial College Students Innovation and Entrepreneurship Training Program Project (grant no. 201810570039). 


\section{Availability of data and materials}

The datasets analyzed during the current study download from Surveillance Epidemiology and End Results database (https://seer.cancer.gov/) and The Cancer Genome Atlas (TCGA) database. (https://cancergenome.nih.gov/).

\section{Authors' contributions}

Zhaohui He, Fucai Tang and Zechao Lu conceived the study; Zhaohui He and Fucai Tang conducted the work; Chengwu He, Hanbin Zhang and weijia Wu obtaining and analyzed the data; Fucai Tang and Zechao Lu wrote the manuscript, and Zhaohui He reviewed the manuscript. All the authors listed have read and approved the manuscript.

\section{Ethics approval and consent to participate}

The present study, the data download from Surveillance Epidemiology and End Results database and The Cancer Genome Atlas (TCGA) database, therefore, this article does not contain any studies with human participants or animals performed by any of the authors. Thus no ethical approval and patient consent are required. This article does not contain any studies with animals performed by any of the authors.

\section{Conflicts of Interests}

All authors declare that they have no conflict of interest to state.

\section{Consent for publication}

Not applicable.

\section{References}

1 Ridge CA, Pua BB, Madoff DC. Epidemiology and staging of renal cell carcinoma. Semin Intervent Radiol. 2014; 31:3-8.

2 Liu K, Wang P, Zhu X, et al. Disparities of age-based cancer-specific survival improvement with various clinicopathologic characteristics for kidney cancer. Cancer Manag Res. 2018; 10:2259-2268. 
3 Takada S, Namiki M, Takahara S, et al. Serum HGF levels in acute renal rejection after living related renal transplantation. Transpl Int. 1996; 9:151-4.

4 Meehan B, Appu S, St Croix B, et al. Age-related properties of the tumour vasculature in renal cell carcinoma. BJU Int. 2011; 107:416-24.

5 Al-Janabi O, Wach S, Nolte E, et al. Piwi-like 1 and 4 gene transcript levels are associated with clinicopathological parameters in renal cell carcinomas. Biochim Biophys Acta. 2014; 1842:686-90.

6 Yusim I, Mermershtain W, Neulander E, et al. Influence of age on the prognosis of patients with renal cell carcinoma (RCC). Onkologie. 2002; 25:548-50.

7 Camp RL, Dolled-Filhart M, Rimm DL. X-tile: a new bio-informatics tool for biomarker assessment and outcome-based cut-point optimization. Clin Cancer Res 2004; 10:7252-9.

8 Taccoen X, Valeri A, Descotes $\mathrm{JL}$, et al. Renal cell carcinoma in adults 40 years old or less: young age is an independent prognostic factor for cancer-specific survival. Eur Urol. 2007; 51:980-7.

9 Aziz A, May M, Zigeuner R, et al. Do young patients with renal cell carcinoma feature a distinct outcome after surgery? A comparative analysis of patient age based on the multinational CORONA database. $J$ Urol. 2014; 191:310-5.

10 Kang HW, Seo SP, Kim WT, et al. Impact of Young Age at Diagnosis on Survival in Patients with Surgically Treated Renal Cell Carcinoma: a Multicenter Study. J Korean Med Sci. 2016; 31:1976-1982.

$11 \mathrm{Kim}$ JH, Park YH, Kim YJ, et al. Is there a difference in clinicopathological outcomes of renal tumor between young and old patients? A multicenter matched-pair analysis. Scand J Urol. 2016; 50:387-91.

12 Komai Y, Fujii Y, limura Y, et al. Young age as favorable prognostic factor for cancer-specific survival in localized renal cell carcinoma. Urology. 2011; 77:842-7.

13 Jung EJ, Lee HJ, Kwak C, et al. Young age is independent prognostic factor for cancer-specific survival of low-stage clear cell renal cell carcinoma. Urology. 2009; 73:137-41.

14 Malouf GG, Camparo P, Oudard S, et al. Targeted agents in metastatic Xp11 translocation/TFE3 gene fusion renal cell carcinoma (RCC): a report from the Juvenile RCC Network. Ann Oncol. 2010; 21:1834-8.

15 Mitchell TJ, Turajlic S, Rowan A, et al. Timing the Landmark Events in the Evolution of Clear Cell Renal Cell Cancer: TRACERx Renal. Cell. 2018; 173:611-623.

16 Malouf GG, Camparo P, Molinié V, et al. Transcription factor E3 and transcription factor EB renal cell carcinomas: clinical features, biological behavior and prognostic factors. J Urol. 2011; 185:24-9.

17 Abbott GW. KCNE4 and KCNE5 K(+) channel regulation and cardiac arrhythmogenesis. Gene. 2016; 593:249-60. 
18 Arabzadeh A, Troy TC, Turksen K. Role of the Cldn6 cytoplasmic tail domain in membrane targeting and epidermal differentiation in vivo. Mol Cell Biol. 2006;26:5876-5887.

19 Yang M, Li Y, Shen X, Ruan Y, Lu Y, Jin X, Song P, Guo Y, Zhang X, Qu H, et al. CLDN6 promotes chemoresistance through GSTP1 in human breast cancer. J Exp Clin Cancer Res. 2017;36:157.

20 Guo Y, Lin D, Zhang M, et al. CLDN6-induced apoptosis via regulating ASK1-p38/JNK signaling in breast cancer MCF-7 cells. Int J Oncol. 2016;48:2435-2444.

21 Gao F, Li M, Xiang R, Zhou X, Zhu L, Zhai Y. Expression of CLDN6 in tissues of gastric cancer patients: Association with clinical pathology and prognosis. Oncol Lett. 2019;17(5):4621-4625

22 Lin Z, Witschas K, Garcia T, et al. A critical GxxxA motif in the gamma6 calcium channel subunit mediates its inhibitory effect on Cav3.1 calcium current. J Physiol. 2008; 586:5349-66.

23 Lee JS, Kim JH, Bae JS, et al. Association of CACNG6 polymorphisms with aspirin-intolerance asthmatics in a Korean population. BMC Med Genet. 2010; 11:138.

24 Patel NH, Attwood KM, Hanzly M, et al. Comparative Analysis of Smoking as a Risk Factor among Renal Cell Carcinoma Histological Subtypes. J Urol. 2012; 194:640-6.

25 Kroeger N, Klatte T, Birkhäuser FD, et al. Smoking negatively impacts renal cell carcinoma overall and cancer-specific survival. Cancer. 2012; 118:1795-802.

26 Golabek T, Bukowczan J, Szopinski T, et al. Obesity and renal cancer incidence and mortality-a systematic review of prospective cohort studies. Ann Agric Environ Med. 2016; 23.37-43.

27 Park J, Morley TS, Kim M, et al. Obesity and cancer-mechanisms underlying tumour progression and recurrence. Nat Rev Endocrinol. 2014; 10: 455-465

28 Chow WH, Dong LM, Devesa SS. Epidemiology and risk factors for kidney cancer. Nat Rev Urol 2010; 7.245-57.

29 Colt JS, Schwartz K, Graubard BI, et al. Hypertension and risk of renal cell carcinoma among white and black Americans. Epidemiology. 2011; 22.797-804.

\section{Tables}

Table $1 \square$ The Clinical characteristic baseline of CCRC patients in SEER 


\begin{tabular}{|c|c|c|c|c|c|c|}
\hline \multirow[t]{3}{*}{ Characteristic } & \multicolumn{3}{|c|}{ Before PSM } & \multicolumn{3}{|c|}{ After PSM } \\
\hline & Younger & Older & & Younger & Older & \\
\hline & $(<=53 \mathrm{y})$ & $(>53 \mathrm{y})$ & $\mathrm{P}$ & $(<=53 \mathrm{y})$ & $(>53 \mathrm{y})$ & $\mathrm{P}$ \\
\hline SEX & & & $<0.001$ & & & 0.613 \\
\hline male & 9134 & 18958 & & 9124 & 9165 & \\
\hline female & 5142 & 12740 & & 5142 & 5101 & \\
\hline Race & & & $<0.001$ & & & 0.322 \\
\hline Black & 1020 & 1939 & & 1014 & 1063 & \\
\hline White & 12199 & 27428 & & 12195 & 12105 & \\
\hline Other & 1057 & 2331 & & 1057 & 1098 & \\
\hline Marital & & & $<0.001$ & & & 0.739 \\
\hline Single/Other & 5124 & 10484 & & 5116 & 5143 & \\
\hline married & 9152 & 21214 & & 9150 & 9123 & \\
\hline SIZE & & & $<0.001$ & & & 0.571 \\
\hline $\operatorname{Dmax}<=4 \mathrm{~cm}$ & 7058 & 12966 & & 7052 & 6981 & \\
\hline $4 \mathrm{~cm}<$ Dmax $<=7 \mathrm{~cm}$ & 3771 & 10175 & & 3771 & 3847 & \\
\hline Dmax $>7 \mathrm{~cm}$ & 3447 & 8557 & & 3443 & 3438 & \\
\hline GRADE & & & $<0.001$ & & & 0.566 \\
\hline [ & 2204 & 4226 & & 2199 & 2243 & \\
\hline ૧ & 7893 & 16362 & & 7890 & 7873 & \\
\hline प & 3376 & 8978 & & 3375 & 3309 & \\
\hline प & 803 & 2132 & & 802 & 841 & \\
\hline Laterality & & & 0.228 & & & 0.840 \\
\hline Left & 6949 & 15622 & & 6946 & 6963 & \\
\hline Right & 7327 & 16076 & & 7320 & 7303 & \\
\hline SEER historic stage A & & & $<0.001$ & & & 0.754 \\
\hline Localized & 11405 & 21988 & & 11395 & 11345 & \\
\hline Regional & 1865 & 6576 & & 1865 & 1892 & \\
\hline Distant & 1006 & 3134 & & 1006 & 1029 & \\
\hline Radiation & & & 0.035 & & & 0.202 \\
\hline NO & 13967 & 30909 & & 13960 & 13928 & \\
\hline YES & 309 & 789 & & 306 & 338 & \\
\hline chemotherapy & & & $<0.001$ & & & 0.274 \\
\hline $\mathrm{NO}$ & 13664 & 29986 & & 13655 & 13617 & \\
\hline YES & 612 & 1712 & & 611 & 649 & \\
\hline
\end{tabular}

Table 2₫cox regression analysis of overall survival 


\begin{tabular}{llll}
\hline Characteristic & Univariate cox & Multivariate cox & Multivariate cox \\
& regression before & regression before & regression after \\
& PSM & PSM & PSM
\end{tabular}

\begin{tabular}{llllll}
\hline $95 \% \mathrm{CI}$ & $\mathrm{P}$ & $95 \% \mathrm{CI}$ & $\mathrm{P}$ & $95 \% \mathrm{CI}$ & $\mathrm{P}$ \\
\hline
\end{tabular}

Age

young

old

Sex

male

female

Race

Black

White

Other

$0.798(0.721-0.884)$

Reference

0.895(0.831-0.964)

0.003

Reference

0.836ロ0.776-0.901ם

$<0.001$

0.795ロ0.722-

0.876 [

$0.910 \square 0.859$

$0.964 \square$

Reference

2.1287

$<0.001$

Reference

$<0.001$

0.001

$<0.001$

Marital

Single/Other

married

Reference

$0.780(0.751-0.811)<0.001$

Reference

0.729ロ0.701-0.758凸

$<0.001$

Reference

$0.711 \square 0.674$ -

$0.750 \square$

$<0.001$

\section{Size}

Dmax $<=4 \mathrm{~cm}$

Reference

1.967(1.868-2.070)

$4 \mathrm{~cm}<$
$\operatorname{Dmax}<=7 \mathrm{~cm}$

Dmax $>7 \mathrm{~cm}$

$<0.001$

$1.849 \square 1.748-1.956 \square$

$1.403 \square 1.330-1.480 \square$

$<0.001$

$<0.001$

\section{Reference}

$\begin{array}{ll}<0.001 & 1.382 \square 1.284- \\ & 1.488 \square\end{array} \quad<0.001$

1.965ロ1.823-

$<0.001$

$1.8491 .748-1.956$

\section{GRADE}

]

Reference

Reference

1.151凸1.081-1.225ロ <0.001

0.966ロ0.907-1.029ロ

0.283

Reference

$2.225 \square 2.086-2.373 \square \quad<0.001$

1.213ロ1.133-1.298凸

$<0.001$

0.988ロ0.9071.075

0.773

$1.287 \square 1.172-$
$1.412 \square$
$<0.001$ 
Laterality

Left

Right

$$
<0.001
$$

Reference

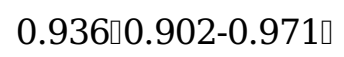

$<0.001$

0.953ロ0.918-0.989ロ

Reference

0.012

$0.947 \square 0.899$ $0.997 \square$

\section{SEER historic} stage A

Localized

Regional

Distant

$11.243 \square 10.734-$ 11.776ロ

Reference

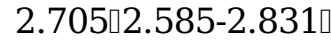

$<0.001$

$<0.001$

5.996(5.631-6.383)

Reference

1.830(1.740-1.925)

$<0.001 \quad 1.860 \square 1.727-$ 2.003 ]

6.694ロ6.115$7.329 \square$

Reference

Reference

YES

6.150ロ5.725-6.607ロ <0.001
$<0.001$ $<0.001$

1.319ロ1.1871.465

Reference

$<0.001$
Reference

5.959ロ5.637-6.299ロ
Reference

$<0.001 \quad 1.254 \square 1.144-$ $1.375 \square$

Table 3־cox regression analysis of cancer-specific survival 


\begin{tabular}{llll}
\hline Characteristic & Univariate cox & Multivariate cox & Multivariate cox \\
& regression before & regression before & regression after \\
& PSM & PSM & PSM
\end{tabular}

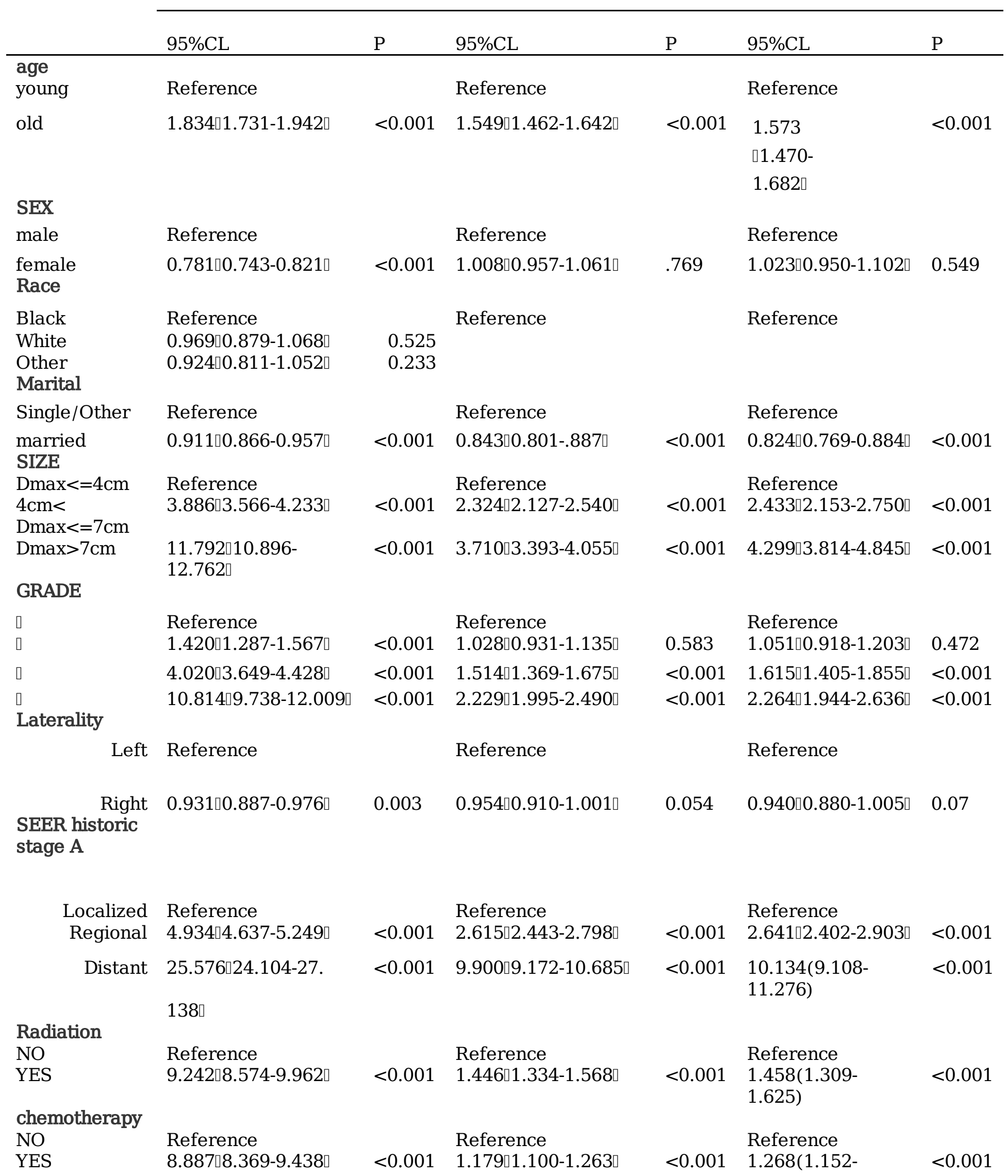


Table $4 \square$ The top 10 age-related differentially expressed genes

\begin{tabular}{lllll}
\hline Gene & \multicolumn{1}{c}{$\operatorname{logFC}$} & \multicolumn{1}{c}{$\log C P M$} & \multicolumn{1}{c}{ PValue } & FDR \\
\hline KCNE5 & -3.871875697 & -0.630531674 & $3.81 E-101$ & $6.88 E-97$ \\
PMEL & -3.118739088 & 3.201080324 & $6.07 E-93$ & $5.48 E-89$ \\
LPAR3 & -3.721054563 & -0.404306233 & $2.46 E-62$ & $1.48 E-58$ \\
SPOCK3 & -4.171219051 & -0.519518204 & $1.49 E-55$ & $6.71 E-52$ \\
PRSS38 & -6.050624343 & -1.293512652 & $9.29 E-54$ & $3.36 E-50$ \\
PAX9 & -2.898326355 & 0.312666906 & $8.33 E-52$ & $2.51 E-48$ \\
LGI3 & -3.236037193 & 0.023278558 & $2.20 E-46$ & $5.67 E-43$ \\
CACNG6 & -3.199514488 & -2.392203888 & $4.55 E-43$ & $1.03 E-39$ \\
CLDN6 & -2.386529645 & -2.048179057 & $1.76 E-42$ & $3.54 E-39$ \\
ALB & 5.560665626 & 6.78720187 & $1.14 E-40$ & $2.06 E-37$ \\
\hline
\end{tabular}

\section{Figures}
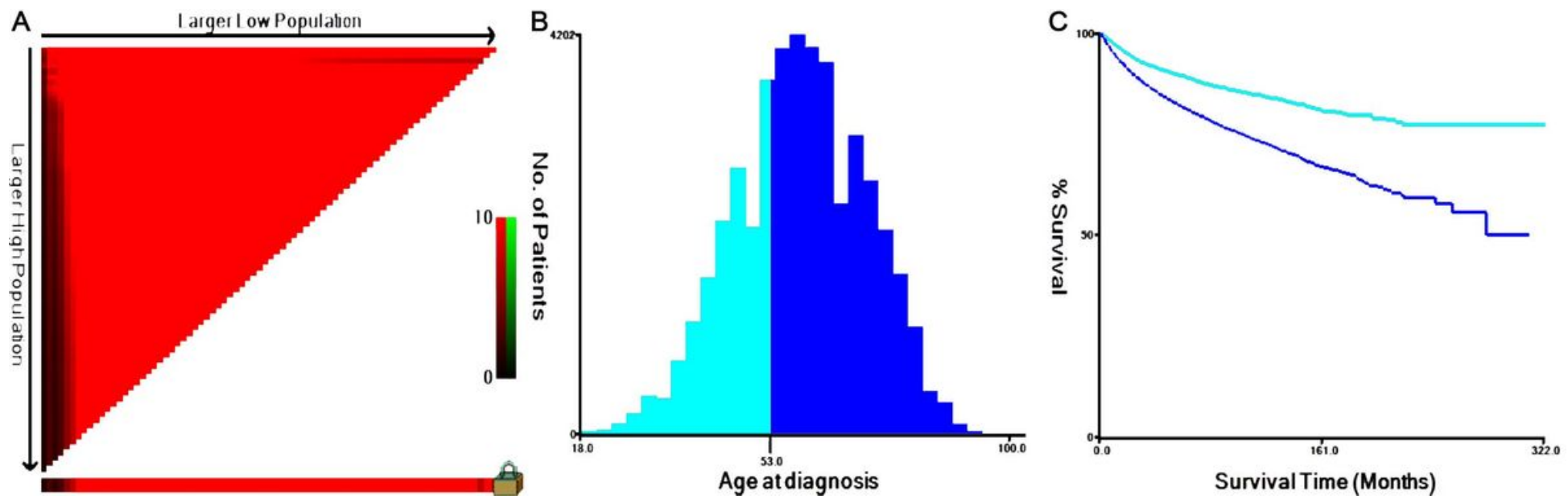

Figure 1

X-Tile Analysis of Survival Data From the Surveillance, Epidemiology, and End Results (SEER) Registry. XTile Analysis Was Performed Using Patient Data From the SEER Registry. The Optimal Cutoff Value Highlighted by the Black Circle in the Left Panel (A) Is Shown on a Histogram of the Entire Cohort (B) and Kaplan-Meier analysis $(C)$ were developed based on these cutoff values. Optimal cut-off values of age at diagnosis were identified as 53 years based on CSS. 
A

OS curve before PSM

Strata + group=old + group=young
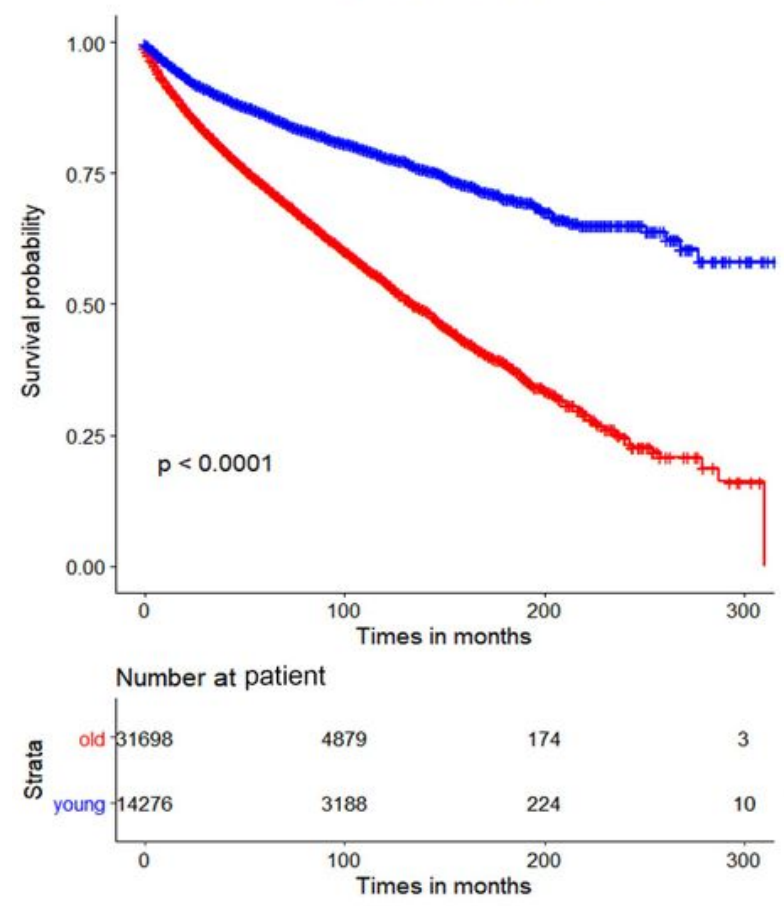

C

OS curve after PSM

Strata + group=old + group=young
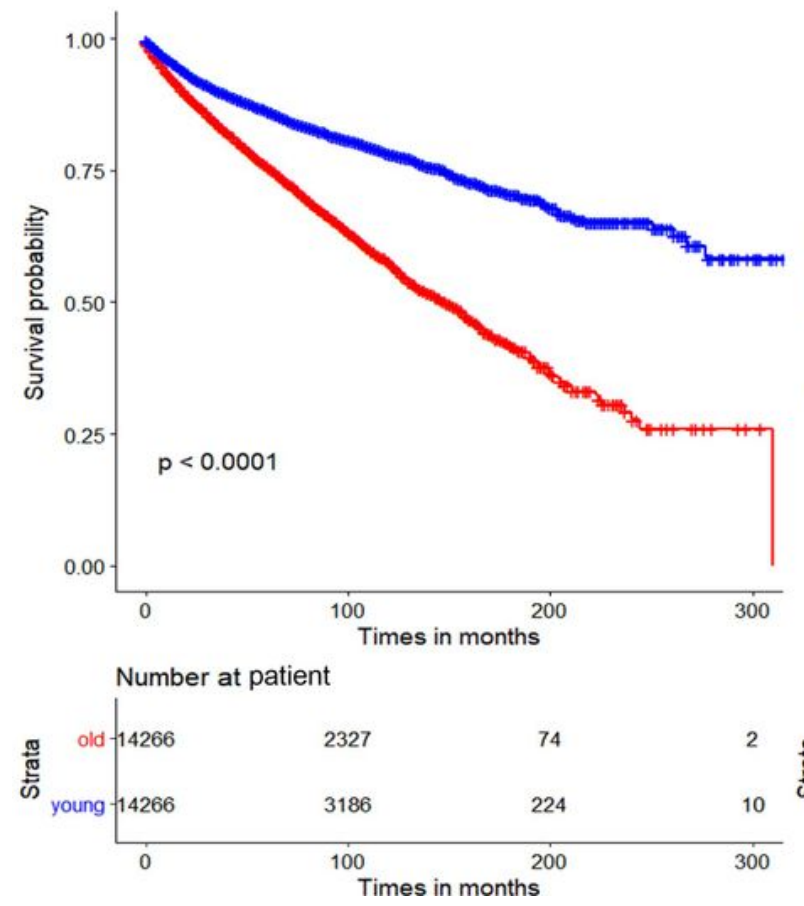

B

CSS curve before PSM

Strata + group=old + group=young
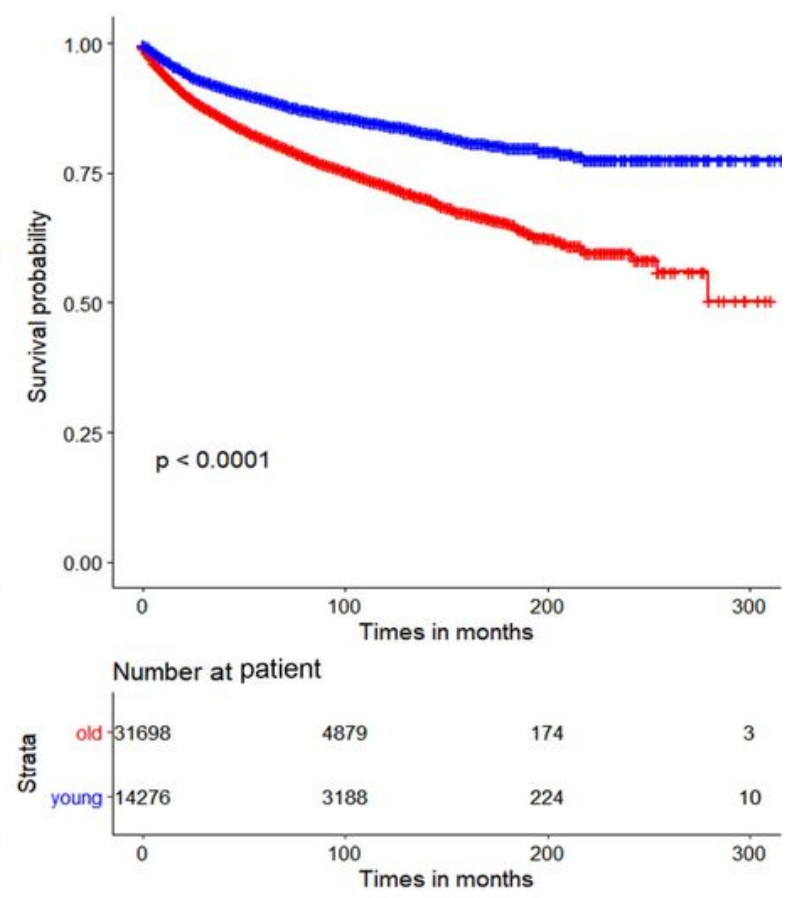

D

CSS curve after PSM

Strata + group=old + group=young

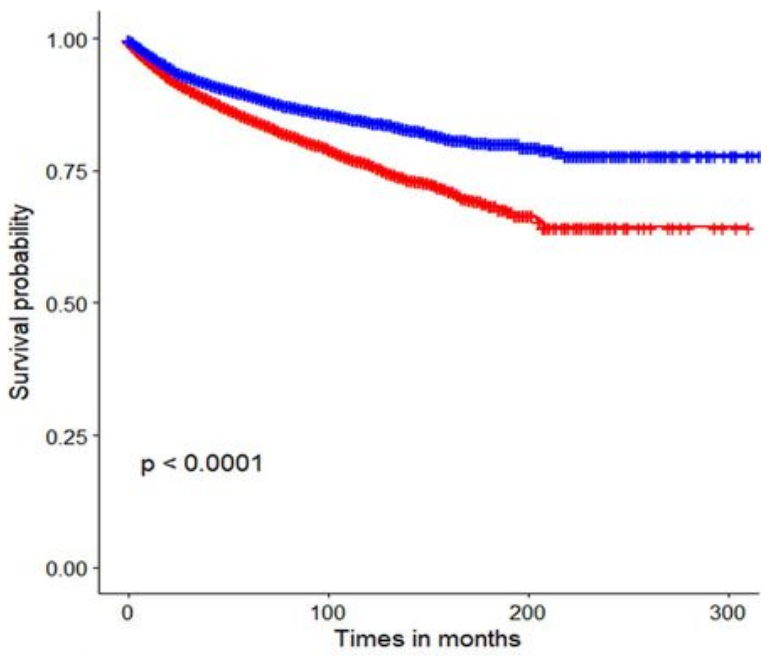

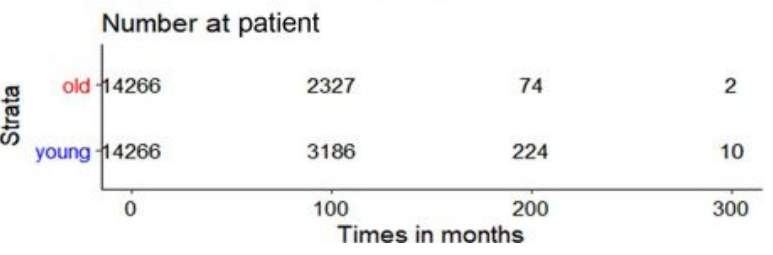

\section{Figure 2}

The Overall Survial (OS) and Cancer-Specific Survival (CSS) Curve of Younger and Older Patients. (A) KaplaneMeier Survival Curve of OS Using Unadjusted Data in the Surveillance, Epidemiology, and End Results Data Set. The 5- and 10-Year OS Rates Were $86.4 \%$ and $78.2 \%$, Respectively, and $72.8 \%$ and $54.5 \%$, Respectively $(\mathrm{P}<0.001)$. (B) KaplaneMeier Survival Curve of CSS Using Unadjusted Data in the Surveillance, Epidemiology, and End Results Data Set. The 5- and 10-Year OS Rates Were $89.4 \%$ and 
81.7\%, Respectively, and $84.3 \%$ and $72.8 \%$, Respectively $(P<0.001)$.(C) KaplaneMeier Survival Curve of OS Using Propensity Score Matching Adjusted Data Set. The Hazard Ratios of OS Using Univariate Analysis in the Matched Cohort Were 2.056(95\% Cl,1.948-2.170; $\mathrm{P}<0.001)$. (D) KaplaneMeier Survival Curve of CSS Using Propensity Score Matching Adjusted Data Set. The Hazard Ratios of OS Using Univariate Analysis in the Matched Cohort Were 1.496(95\% Cl,1.399-1.600; $\mathrm{P}<0.001)$. The $\mathrm{P}$ Values Were Calculated Using the Cox Model.

\section{Subgroup analysis for the CCRCC Patients in SEER}

\section{Subgroup}

Overall

Sex

male

female

Race

Black

White

Other

\section{Marital status}

Single/Other

Married

\section{Size}

Dmax $<=4 \mathrm{~cm}$

4< Dmax $<=7 \mathrm{cn}$

Dmax $>7 \mathrm{~cm}$

SEER Grade

Grade 1

Grade 2

Grade 3

Grade 4

Laterality

Left

Right

SEER historic stage A

Localized

Regional

Distant

Radiation

NO

YES

Chemotherapy

NO

YES
Hazard Ratio(95\%) P Value $1.834(1.731-1.942) \quad<0.001$

$1.742(1.627-1.864) \quad<0.001$

$2.176(1.953-2.424) \quad<0.001$

$1.584(1.283-1.956) \quad<0.001$

$1.860(1.748-1.979) \quad<0.001$

$1.795(1.445-2.232) \quad<0.001$

$1.775(1.614-1.953) \quad<0.001$

$1.876(1.745-2.016) \quad<0.001$

3.710(3.006-4.579) $\quad<0.001$

$2.171(1.926-2.447) \quad<0.001$

$1.399(1.305-1.500) \quad<0.001$

3.158(2.481-4.022) $<0.001$

2.607(2.339-2.905) $<0.001$

$1.350(1.238-1.473) \quad<0.001$

$1.138(1.007-1.286)$

$1.733(1.600-1.877) \quad<0.001$

$1.939(1.786-2.106) \quad<0.001$

$2.412(2.158-2.695) \quad<0.001$

$1.210(1.077-1.359) \quad 0.001$

1.114(1.022-1.214) $\quad 0.014$

1.949(1.832-2.073)

$1.028(0.881-1.200)$

2.005(1.878-2.142)

1.095(0.972-1.233)

$<0.001$

0.724

$<0.001$

0.135
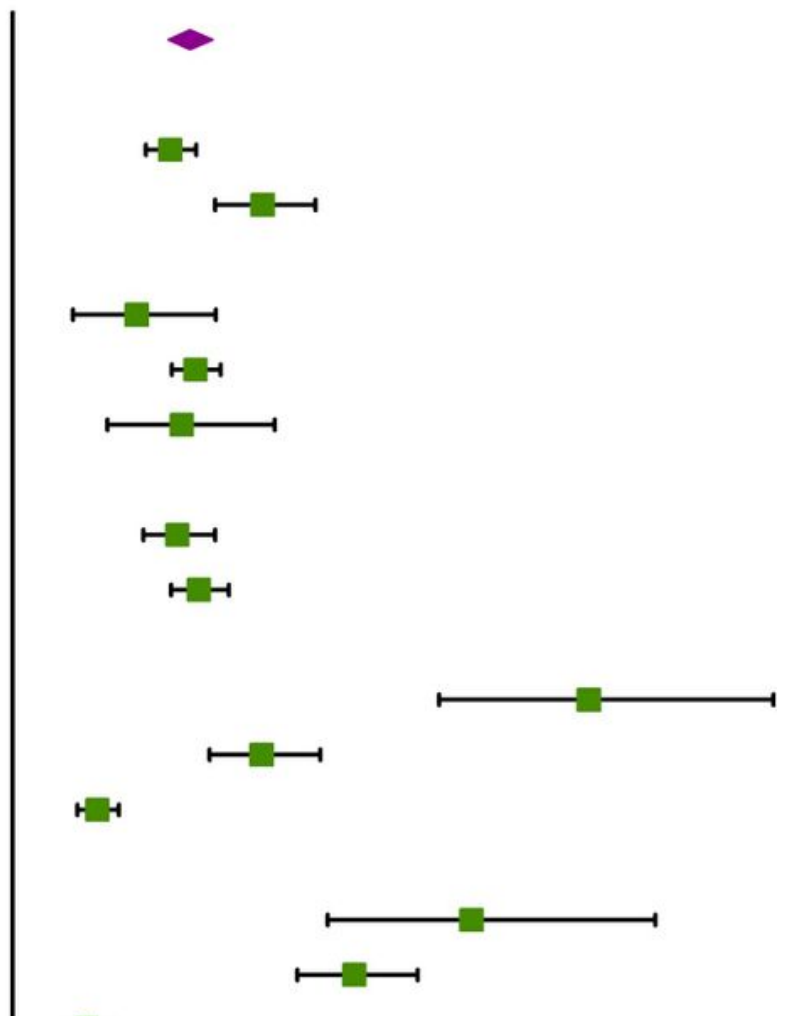

느멉

$-$

$\mapsto$ 는
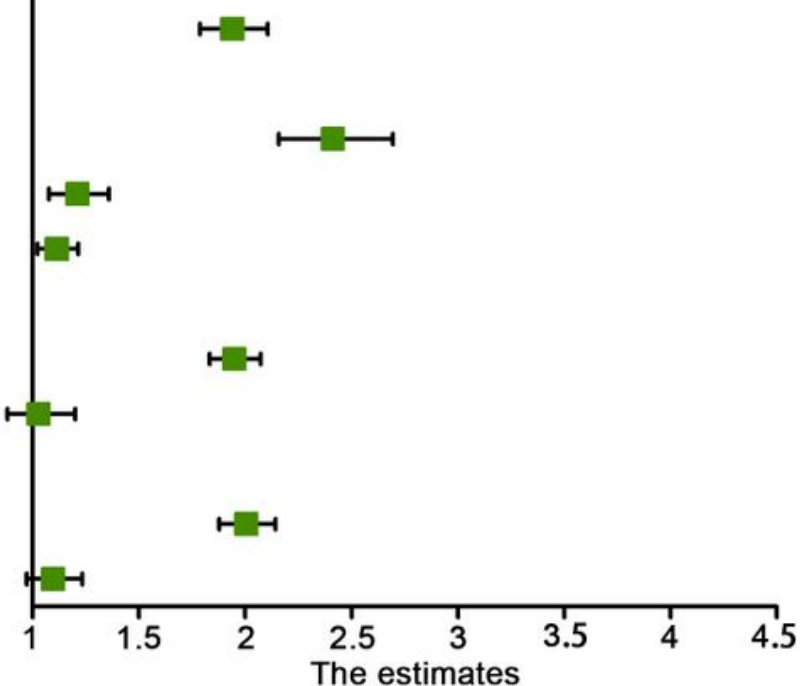

Figure 3 
Subgroup analysis according to variable and the probability of CCRCC cancer-specific survival analysis according to age (Young group vs. Older group) in the SEER data set. The subgroup analyses were performed according to sex, race, marital status, size, grade, laterality, SEER historic stage A, radiation, and chemotherapy.
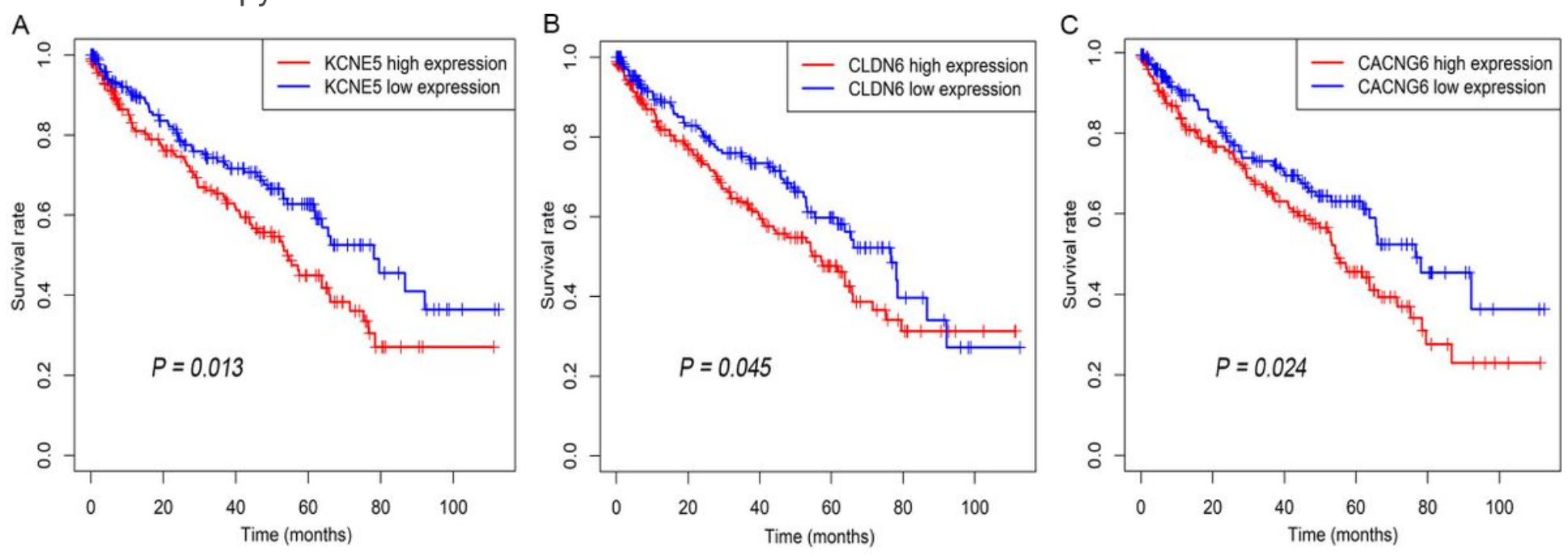

Figure 4

Survival analysis of top10 gene from the DEGs. A: Kaplan-Meier analysis were developed based on expression level of KCNE5 ( $p=0.013)$; B: Kaplan-Meier analysis were developed based on expression level of CLDN6 ( $p=0.045)$; C: Kaplan-Meier analysis were developed based on expression level of CACNG6 $(p=0.024)$.

\section{Supplementary Files}

This is a list of supplementary files associated with this preprint. Click to download.

- supplement1.docx 